\title{
Errata: Imaging of glioma tumor with endogenous fluorescence tomography
}

Dax S. Kepshire, ${ }^{\text {a }}$ Summer L. Gibbs-Strauss, ${ }^{a}$ Julia A. O'Hara, ${ }^{\text {a,d }}$ Michael Hutchins, ${ }^{\text {b Niculae Mincu, }}{ }^{\text {b }}$ Frederic Leblond, ${ }^{a}$ Mario Khayat, ${ }^{\mathrm{b}}$ Hamid Dehghani, Subhadra Srinivasan, ${ }^{a}$ and Brian W. Pogue ${ }^{\mathrm{a}}$

a Dartmouth College, Thayer School of Engineering, Hanover, New Hampshire 03755

${ }^{\mathrm{b}}$ ART Advanced Research Technologies Inc., Saint-Laurent, Quebec, Canada H4S 2A4

'University of Exeter, School of Physics, Stocker Road, Exeter, EX4 4QL, United Kingdom

${ }^{\mathrm{d}}$ Dartmouth Medical School, Department of Diagnostic Radiology, Hanover, New Hampshire 03755 [DOI: $10.1117 / 1.3157198]$

This article [J. Biomed. Opt. 14, 030501 (May/June 2009)] was originally published online on 11 May 2009 with an error in the spelling of an author name. The name Gibbs-Struass was corrected to Gibbs-Strauss. All online versions of the article were corrected on 20 May 2009 and the article appears correctly in print. 